\title{
Exploring the relationship between environmental enteric dysfunction and oral vaccine responses
}

\author{
James A Church*,1,2, Edward PK Parker ${ }^{3}$, Margaret N Kosek ${ }^{4}$, Gagandeep Kang ${ }^{5}$, Nicholas \\ C Grassly ${ }^{3}$, Paul Kelly ${ }^{2,6}$ \& Andrew J Prendergast ${ }^{1,2,4}$ \\ ${ }^{1}$ Zvitambo Institute for Maternal \& Child Health Research, Harare, Zimbabwe \\ ${ }^{2}$ Centre for Genomics \& Child Health, Blizard Institute, Queen Mary University of London, UK \\ ${ }^{3}$ Department of Infectious Disease Epidemiology, St Mary's Campus, Imperial College London, London, UK \\ ${ }^{4}$ Department of International Health, Johns Hopkins Bloomberg School of Public Health, Baltimore, MD 21205, USA \\ ${ }^{5}$ Department of Gastrointestinal Sciences, Christian Medical College, Vellore, Tamil Nadu, India \\ ${ }^{6}$ Tropical Gastroenterology \& Nutrition group, University of Zambia School of Medicine, Lusaka, Zambia \\ *Author for correspondence: j.church@qmul.ac.uk
}

Oral vaccines significantly underperform in low-income countries. One possible contributory factor is environmental enteric dysfunction (EED), a subclinical disorder of small intestinal structure and function among children living in poverty. Here, we review studies describing oral vaccine responses and EED. We identified eight studies evaluating EED and oral vaccine responses. There was substantial heterogeneity in study design and few consistent trends emerged. Four studies reported a negative association between EED and oral vaccine responses; two showed no significant association; and two described a positive correlation. Current evidence is therefore insufficient to determine whether EED contributes to oral vaccine underperformance. We identify roadblocks in the field and future research needs, including carefully designed studies those can investigate this hypothesis further.

First draft submitted: 12 January 2018; Accepted for publication: 26 March 2018; Published online: 21 June 2018

Keywords: EED • infant • oral vaccine

Although the last decade has witnessed impressive declines in the global burden of diarrheal disease [1], it remains the second largest infectious cause of child mortality with 578,000 child deaths annually [2]. Furthermore, diarrhea causes extensive morbidity, particularly in young children, with an estimated 957.5 million episodes of disease and 7506 thousand years lost due to ill health in 2016 [3].

A major contributor to the global reduction in diarrhea has been improved coverage of oral rotavirus vaccination, which had been introduced in 93 countries by November 2017 [4]. Oral vaccination is also a key prevention strategy against enteric bacterial pathogens such as Vibrio cholerae and Salmonella typhi; and vaccines against other bacteria including Shigella and Campylobacter are in development. Meanwhile the sustained role of oral poliovirus vaccine (OPV) in the polio endgame strategic plan further emphasizes the potential impact of oral vaccines in mitigating enteric infections. Oral vaccines obviate the need for needles, are easy to administer and generate a local immune response in the gut, thereby conferring protection and reducing the likelihood of onward transmission. However, the success of two of the available oral vaccines (rotavirus and polio) also hides a troublesome truth: they significantly underperform in low-income compared with high- and middle-income countries [5].

Stanley Plotkin noted as early as 1960 that Congolese infants were less likely to develop neutralizing antibodies to an attenuated type 1 poliovirus vaccine compared with counterparts in Europe and the USA [6]. Seroconversion to OPV has remained consistently lower in low-income compared with developed countries [7], which has hampered global eradication efforts. Poor immunogenicity has also been reported for both live and killed oral cholera vaccines [8]. Finally, oral vaccine efficacy against severe rotavirus gastroenteritis is substantially lower in sub-Saharan Africa (39.3\%, 95\% CI: 19.1-54.7) [9] and Asia (48.3\%, 95\%CI: 22.3-66.1) [10] than in Europe/USA (8598\%) $[11,12]$.

Future Medicine 
Box 1. Outstanding research questions.

- Does environmental enteric dysfunction (EED) reduce oral vaccine performance?

- Can prevention or treatment of EED improve responses to oral vaccines?

- Are some oral vaccines more susceptible than others to EED?

- What is the interplay between EED, diarrhea, enteropathogen carriage, composition of the microbiota and underperformance of oral vaccines?

- Would an EED point of care test identify children at higher risk of oral vaccine failure?

Although a number of factors have been proposed to explain this efficacy gap, the precise biological mechanisms have not been fully elucidated [13]. Notably, parenteral vaccines do not display diminished efficacy in these same contexts suggesting that systemic immunodeficiencies are not underlying these attenuated responses. As a result, factors associated with the intestinal milieu have been proposed as critical determinants. These include interference from maternally acquired antibodies [14], early infection with enteropathogens [15], glycans expressed on the gut epithelium [16], micronutrient and macronutrient deficiencies [17], intestinal dysbiosis [18], aflatoxin exposure [19] and a subclinical disorder of small intestinal structure and function termed environmental enteric dysfunction (EED) [20]. In this review, we critically evaluate the evidence supporting the role of EED in reducing oral vaccine immunogenicity (Box 1) and highlight the current research gaps and future opportunities.

\section{Environmental enteric dysfunction}

EED is a subclinical condition affecting the small bowel. It is almost ubiquitous among children living in poverty, among whom oral vaccines have consistently underperformed [20]. It is characterized morphologically by the presence of intestinal villous flattening, crypt hyperplasia and a chronic T-cell-mediated inflammatory enteropathy [21,22]. Structural changes are accompanied by functional disturbances of increased intestinal permeability and reduced absorptive capacity [23-25]. EED was first reported many decades ago as an incidental finding on small intestinal biopsies (then termed 'tropical enteropathy') [26], but has garnered renewed interest in recent years, with researchers seeking to better define its pathogenesis and consequences and to evaluate preventive or curative interventions.

The etiology of EED remains elusive. Intestinal biopsies taken from stillborn fetuses indicate that EED is not present at birth [27] but rather develops early in infancy and appears to persist into adulthood. Studies of expatriates and migrants have shown that EED can be reversed by a change in environment, specifically by transfer from an environment of poor water and hygiene to the USA or Europe [28,29]. Moreover, there is a wealth of observational data supporting a role for microbial exposure in the development and persistence of EED. Children living in conditions of poor hygiene with sustained exposure to feco-oral contamination are more likely to develop EED than children living in less contaminated households [30]. A recent mouse model of microbial exposure and moderate malnutrition, which recapitulates the steps needed to invoke EED, supports this [31]. Children in lowincome countries also experience a high multiplicity of infections even with no diarrhea [15]. However, while specific organisms have been associated with deranged gut biomarkers [32-35], no single pathogen has been implicated to date as singularly important. Instead, it is likely that persistent exposure to multiple enteropathogens is important. Small intestinal bacterial overgrowth (SIBO), which has been correlated with biomarkers of EED in Bangladeshi children [36], occurs in a proportion of children living in impoverished settings, and its relationship to EED remains to be elucidated [37]. In addition to carriage of pathogenic organisms, changes in the commensal microbiota may also play a role. A recent study of 81 Malawian children showed that the fecal microbiota differs across a spectrum of EED severity (specifically three categories of gut permeability defined by lactulose:mannitol ratios [38]). The three genera Megasphaera, Mitsuokella and Sutterella were more abundant in severe EED versus no EED while the genera Succinivibrio, Klebsiella and Clostridium XI were less abundant.

A number of obstacles limit our understanding of EED. Consensus on a formal case definition for EED is currently lacking. There are ethical and practical constraints to obtaining small intestinal biopsies from asymptomatic young children; studies therefore tend to rely on a limited range of noninvasive biomarkers to identify alterations in gut structure and function. It has been argued that biomarkers capable of detecting early changes in intestinal inflammation or enterocyte mass could ultimately become more sensitive than direct visualization to measure the condition and how it evolves [39]. Indeed, other chronic intestinal disorders, such as inflammatory bowel disease, employ biomarkers, although these are generally used in conjunction with endoscopic surveillance. In contrast, because biopsies are difficult to obtain in EED, very few corroborative studies have compared noninvasive 
Table 1. Four domains of biomarkers used to measure environmental enteric dysfunction.

\begin{tabular}{|ll}
\hline Domains & Biomarkers \\
\hline Intestinal permeability & $\begin{array}{l}\text { D-xylose, mannitol or rhamnose absorption, lactulose paracellular uptake, } \\
\text { lactulose:mannitol ratio, AAT leakage into gut lumen, zonulin }\end{array}$ \\
\hline Intestinal epithelial damage \& repair & $\begin{array}{l}\text { I-FABP, plasma citrulline and/or conversion of alanyl-glutamine to citrulline, lactose } \\
\text { tolerance test (as marker of brush border damage), fecal lipocalin, Fecal Reg1B } \\
\text { (epithelial cell renewal) }\end{array}$ \\
\hline Intestinal inflammation & Stool calprotectin, MPO, lactoferrin, neopterin \\
\hline Microbial translocation \& systemic immune activation & $\begin{array}{l}\text { Plasma LPS core antibody and/or LPS binding protein, circulating soluble CD14, KT } \\
\text { ratio, plasma cytokines, CRP }\end{array}$ \\
\hline Other & Hydrogen breath testing (as a measure of SIBO) \\
\hline $\begin{array}{l}\text { Biomarkers not detailed in Keusch review table are labeled in italics. } \\
\text { AAT: } \alpha 1 \text {-anti-trypsin; CRP: C-reactive protein; I-FABP: Intestinal fatty acid binding protein; KT ratio: Kynurenine-tryptophan ratio; LPS: Lipopolysaccharide; MPO: Myeloperoxidase; SIBO: } \\
\text { Small intestinal bacterial overgrowth. }\end{array}$ & \\
Adapted from [39]. &
\end{tabular}

biomarkers with histological changes; where it has been done in adult populations, they do not appear to correlate well [40,41].

We do not yet fully understand the exposures that underpin EED, the timing of onset and whether the changes in the small bowel that characterize EED are deleterious or adaptive for young children who are repeatedly exposed to multiple enteric pathogens. We use the term EED throughout this paper although we recognize an ambiguity about whether the condition comprises a 'dysfunction'. Rather than being a distinct condition with a single etiology, EED may be the final common pathway arising from multiple overlapping and interacting insults [20]. A single biomarker will probably never capture this. Similarly, it is unlikely to be biologically relevant to categorize the binary presence or absence of EED; rather, there appears to be a population-level alteration in gut structure and function of severity, which varies over time and appears to be especially important in young children.

\section{EED \& oral vaccine failure}

Several findings have led to speculation that EED may shape geographic trends in oral vaccine underperformance $[42,43]$. First, EED evolves during the period when oral vaccines are administered in early infancy. Second, oral vaccines are more likely to fail in low-income countries [44], where EED is prevalent. Third, oral vaccine failure is prone to seasonal variations [45] in a manner that has also been described for EED [40]. A plausible rationale for $\mathrm{EED}$ as a cause of oral vaccine failure also exists at the biological level. The intestinal mucosa is hugely dynamic, with important functions as a mechanical, antimicrobial and immunological interface. Widespread and profound alterations in gut structure and function may interfere with the processing of an oral vaccine as it transits through the small intestine.

At present, there is broad consensus that a range of EED biomarkers will provide the most informative measure of associations between gut dysfunction and oral vaccine response [46-48]. Four pathological domains have been proposed (Table 1) [39]: intestinal permeability; intestinal epithelial damage and repair; intestinal inflammation; and microbial translocation and immune activation. Incorporating each of these domains into a conceptual framework, we outline mechanisms through which disturbances in these domains might influence oral vaccine 'take' (Figure 1). Intestinal epithelial damage, for example, may alter Peyer's patches and the processing efficiency of the mucosal immune interface. Intestinal inflammation may be characterized by a proinflammatory mucosal response together with increased numbers of immunomodulatory molecules acting as inhibitors, increased numbers of Tregs and enhanced dendritic cell function $[49,50]$. However, the degree to which each domain contributes to the pathogenesis of oral vaccine failure likely varies. In the following section, we evaluate each of these domains of EED in turn and the evidence for their contribution to oral vaccine failure in low-income countries.

\section{Search method}

We conducted a systematic search using Medline (see Appendix 1) for articles published on or before 2 May 2017 describing vaccine responses in the context of EED. Abstracts and titles from all years were compiled in Endnote (Thomson Reuters) and screened. We extracted data on study year, country, participant number and age, vaccine type, choices of EED biomarkers, measurement time points and analysis. The search was subsequently broadened also to include studies detailing oral vaccine responses in the context of other gastrointestinal conditions 


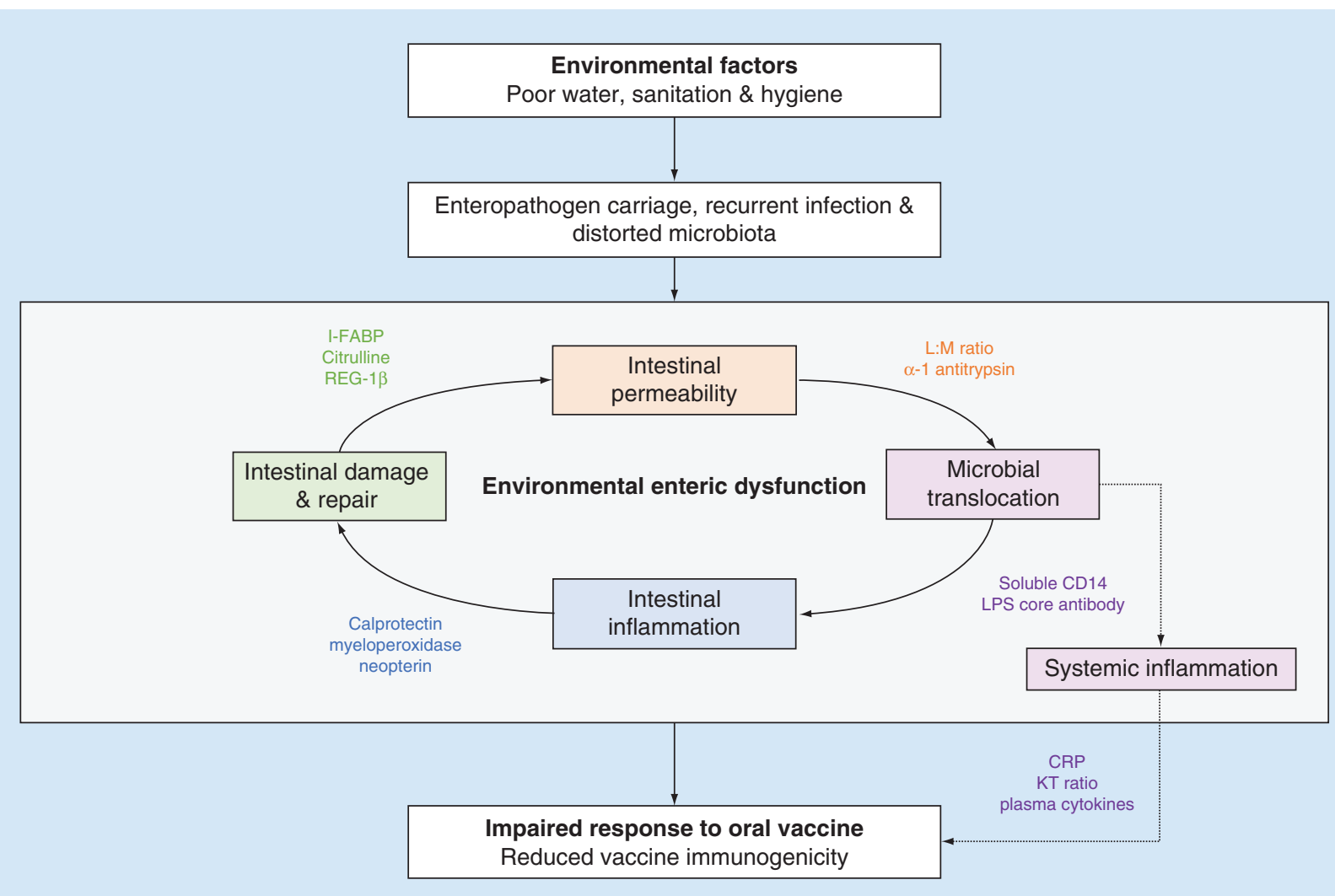

Figure 1. Proposed biological pathway demonstrating the hypothesized role of EED (incorporating four domains depicted by four colours) in the underperformance of oral vaccines.

with phenotypic features in common with EED. Our review was restricted to articles either written in English or translated into English. Unpublished data were not included; however, two studies published during the course of the review were subsequently included.

\section{Results overview}

We identified eight studies describing oral vaccine responses in the context of EED across six countries and three continents (South America, Africa and India). Six studies describe immune responses to OPV and/or rotavirus vaccine (RVV) in infants (under 1 year of age) and two studies focus on responses to oral cholera vaccine in older children aged 3-14 years (Table 2).

These studies used a range of biomarkers to characterize EED, with each selecting a different combination of markers measured at various time points (Table 2). Three studies included at least one biomarker from all four domains. Four of the eight studies reported overall a negative association between EED and oral vaccine responses, with elevated markers of EED associated with reduced oral vaccine performance $[51,53,54,56]$. Two studies showed no significant association [16,52] and two studies concluded that EED was positively correlated with immunogenicity to oral vaccine antigens $[55,57]$. The majority of studies relied on vaccine immunogenicity as a proxy for oral vaccine efficacy. The results of these studies are summarized in Figure 2 (with additional data detailing ranges and effect sizes available in Appendix 2).

\section{Domain 1 - intestinal permeability}

Dual-sugar tests have been regarded as the gold standard for assessing intestinal permeability. They rely on measuring the fractional excretion of nonmetabolizable saccharides of varying sizes (most commonly lactulose and or mannitol) in urine after oral ingestion. The smaller sugar, mannitol, should be absorbed by healthy villi and be detected in the urine, while the larger sugar, lactulose, should not be absorbed due to intact tight junctions between enterocytes. Urinary lactulose recovery, or an elevated lactulose:mannitol ratio, may therefore reflect increased 


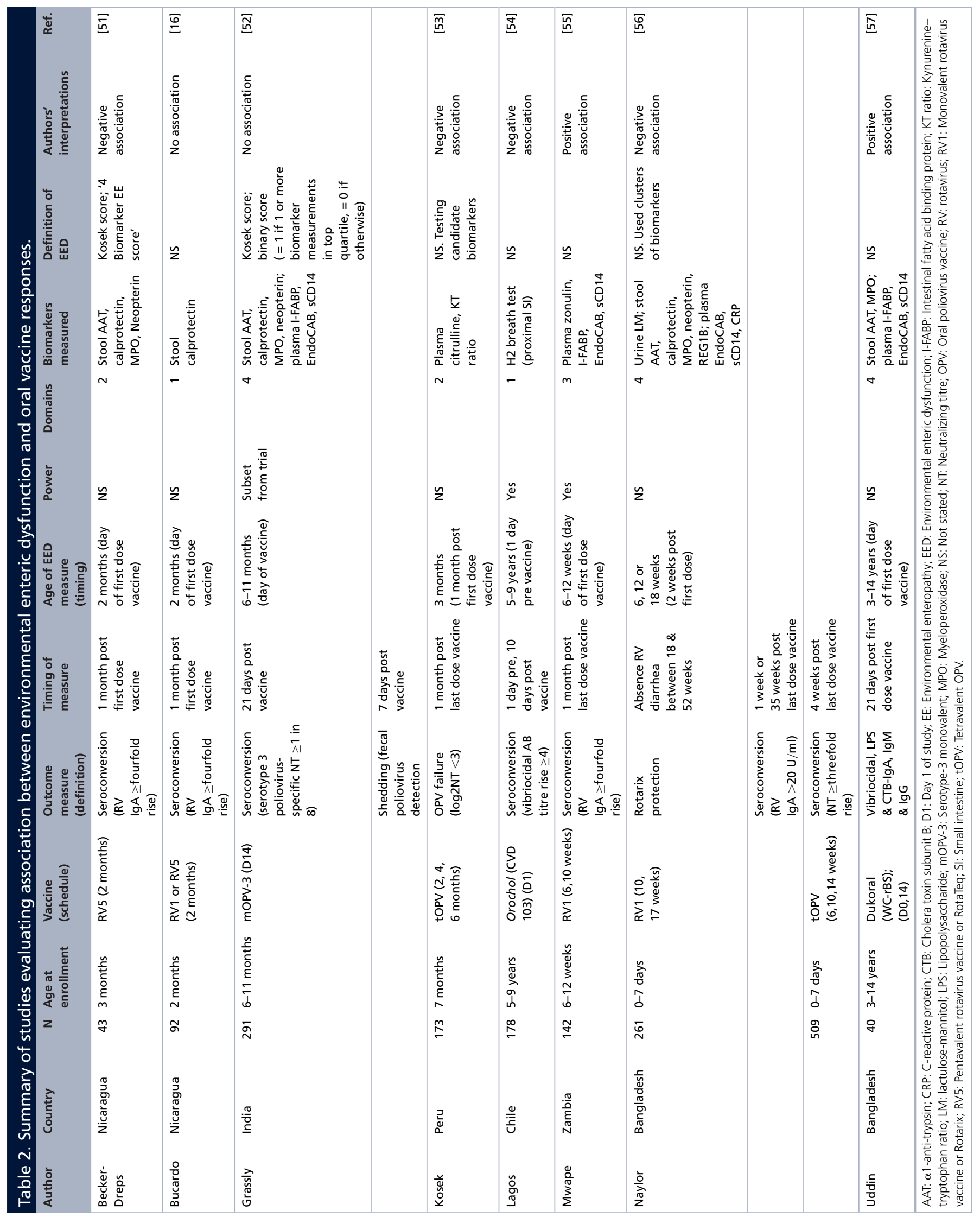




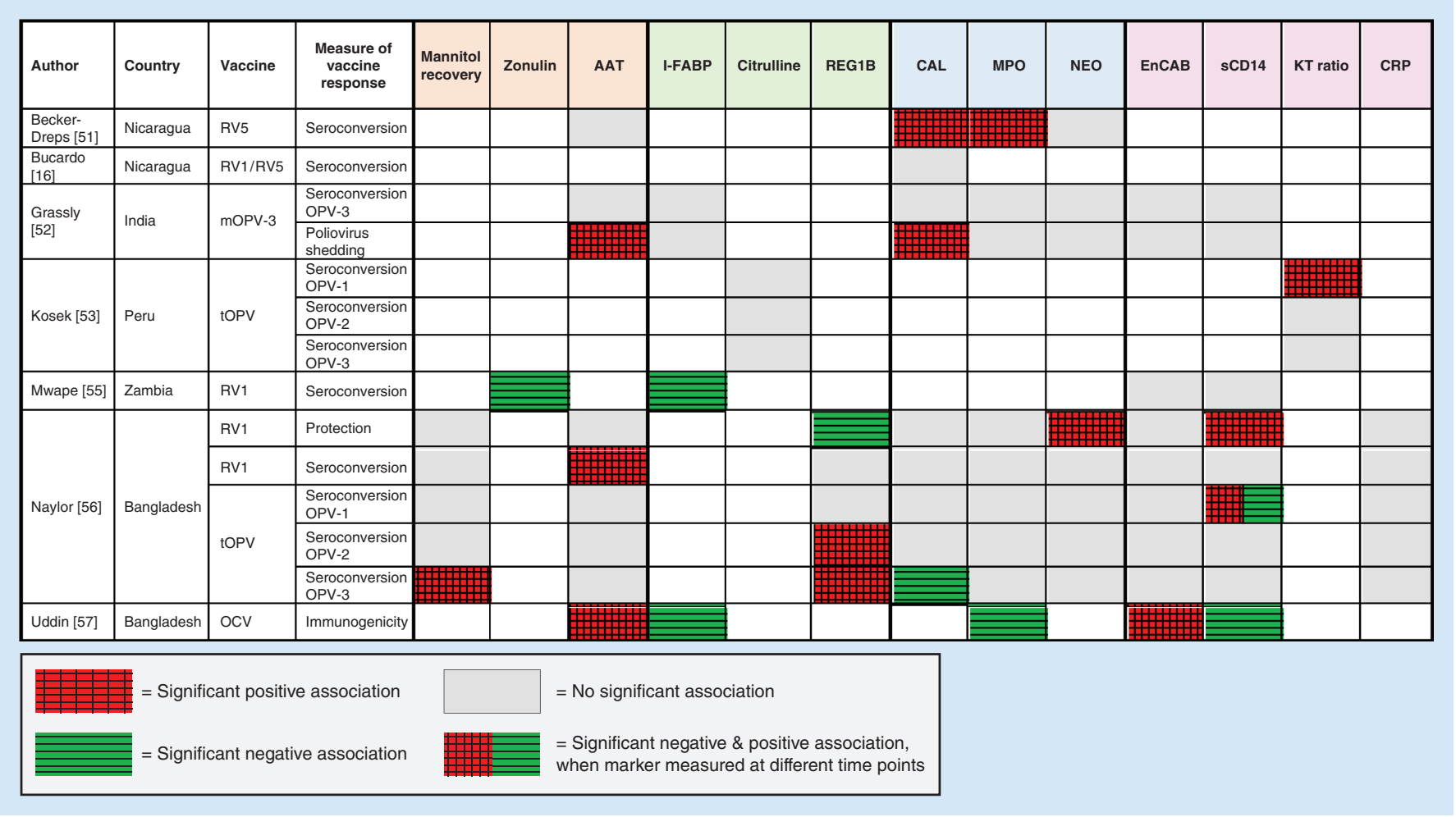

Figure 2. Summary of evidence for association between markers in four domains of environmental enteric dysfunction and oral vaccine response.

intestinal permeability. However, the test has several limitations [58]: first, it is time-consuming, requiring a period of fasting and prolonged urine collection, which is difficult in young infants; second, the ingested solute load may alter intestinal transit time and permeability; and third, variations in test procedure and analysis make cross-study comparisons challenging. Only one study reported a dual-sugar test in relation to oral vaccine responses (Figure 2). In a cohort of 509 Bangladeshi infants, urinary mannitol levels measured at 3 months of age were negatively associated with OPV3 seroconversion [56]; however, no association was found for other strains of OPV, neither with Rotarix immunogenicity nor protection from rotavirus diarrhea.

$\alpha-1$ antitrypsin (AAT) is a plasma protein synthesized by the liver. It is not normally detectable in the stool of healthy subjects and its presence indicates leakage of AAT from the blood to the intestinal tract due to increased gut permeability. However, quantifying the degree of intestinal permeability is problematic because plasma levels of AAT are prone to variation between subjects as it is an acute phase reactant. In the context of vaccine responses, AAT concentration has been the most frequently used marker of gut permeability. Four studies of three different oral vaccines have examined associations between oral vaccine responses and AAT concentrations (Figure 2). Two large studies $(\mathrm{N}>250)$ in Bangladesh and India found no association between seroconversion to OPV and AAT (measured at 3 months of age in Bangladesh and between 6-11 months of age in India). The Bangladesh study reported a negative association between RV1 seroconversion and AAT; however, AAT concentrations did not correlate with Rotarix protection from rotavirus diarrhea (recorded up to the age of 1 year in vaccinated infants). In another study of RotaTeq in Nicaragua there was no association between seroconversion and AAT, although the sample size in this study was small $(\mathrm{N}=43)$ [51]. Finally, in another cohort of older Bangladeshi children, stool AAT was negatively associated with IL-10 (regulatory) T-cell responses specific to cholera toxin $(p=0.02)$ [57]; however, there was no significant association with more widely used measures of cholera vaccine response (vibriocidal antibodies or lipopolysaccharide [LPS] and cholera toxin subunit B [CTB] antibodies).

Zonulin is a protein that binds to specific receptors on intestinal epithelial cells inducing the disassembly of tight junctions and thereby increasing permeability. One study, among Zambian infants, measured zonulin levels at the time of oral vaccine administration and showed a positive association between zonulin and subsequent Rotarix 
seroconversion [55]. Therefore, in contrast to the other four studies showing no or negative associations, this study is the first to propose that increased intestinal permeability may actually enhance oral vaccine seroconversion.

\section{Domain 2 - enterocyte mass \& function}

Several biomarkers reflect damage, repair and mass of the intestinal epithelium. Intestinal fatty acid binding protein (I-FABP), found in mature enterocytes of the small and large intestine, is released into the serum when the cell membrane is compromised [56]. Owing to its short half-life, it is a dynamic measure of acute enterocyte damage but may be less informative of chronic injury. Two studies found a positive association between levels of I-FABP and oral vaccine responses. One with cholera toxin-specific effector memory $\mathrm{T}$ cells in Bangladeshi children, although this association was not observed for other immunological markers of cholera response [57]; the other, with rotavirus IgA in Zambian infants [55]. Together, they suggest that gut epithelial injury can enhance immune responses to oral cholera and rotavirus vaccines, respectively. However, another study measuring I-FABP in Indian children 6-11 months of age, reported no associations with OPV3 seroconversion or shedding (Figure 2) [52].

Regenerating gene 1B (Reg-1B) is a protein measurable in stool, which has been implicated in the regeneration and repair of the intestinal epithelium [56]; and is upregulated in response to enteric infections [59] and inflammatory conditions [60]. Only one study, conducted in Bangladesh, reports Reg-1B in relation to oral vaccine response [56]. Associations with Reg-1B were found in opposite directions for OPV and Rotarix: elevated Reg-1B at 6 and 12 weeks of age was associated with failure to seroconvert to OPV2 and OPV3, respectively, but with protection from rotavirus diarrhea. Despite this, the authors conclude overall that EED is associated with oral vaccine underperformance.

Citrulline is produced mainly by enterocytes in the small bowel and provides a measure of enterocyte mass [61], independent of nutritional status and inflammation [61]. We identified only one study, which found no associations between citrulline and OPV response in 173 Peruvian children [53]. No studies have evaluated plasma zonulin (a biomarker of tight junction function) or fecal lipocalin (a biomarker of intestinal epithelial damage) in the context of oral vaccine performance.

\section{Domain 3 - intestinal inflammation}

Biochemical measures of intestinal inflammation have been widely used in clinical practice and research as diagnostic and surveillance tools for enteric infections and inflammatory bowel disease [62]. Fecal calprotectin and myeloperoxidase (MPO) are both products of neutrophils, and neopterin is a product of macrophages and dendritic cells. The benefit of these biomarkers is that they are robust and easily reproducible with commercially available assays; however, to date there are no reference ranges for these values among children in low-income settings.

Five studies describe levels of gut inflammatory biomarkers in relation to oral vaccine responses (Figure 2). Among 590 Bangladeshi infants aged 3 months in the PROVIDE study, low levels of fecal neopterin were associated with Rotarix success (no rotavirus diarrhea in the year following vaccination) suggesting a detrimental effect of a proinflammatory milieu on vaccine performance or that children who avoided a recent enteric virus infection had a better vaccine take. In the same study, increased calprotectin was associated with successful OPV3 seroconversion [56], which could be consistent with an enhancing effect of intestinal inflammation. However, other studies do not support this and even describe the converse. One study in Nicaragua showed no association between calprotectin and seroconversion to either Rotarix or RotaTeq [16]. In a trial of azithromycin in India, fecal calprotectin was significantly lower among infants who shed poliovirus 7 days after OPV administration (although an association was not found for seroconversion) [52]. Finally, in a different study of Nicaraguan infants, elevated calprotectin at 2 months of age was associated with reduced seroconversion to RotaTeq [57]. Among the same Nicaraguan infants, elevated MPO was also associated with failure to seroconvert to RotaTeq. However, there was no significant association between MPO and OPV3 response in the India trial or with OPV and rotavirus responses in the PROVIDE study [52,56]. Intriguingly, among older children in Bangladesh, elevated MPO was associated with improved responses to an oral cholera vaccine [57].

An alternative strategy when examining associations with EED involves grouping together biomarkers as in prior studies of stunting. The Mal-ED study showed that a combination of three fecal markers outperformed any single marker in predicting linear growth deficits [63]. Similarly, in a cohort of 6-26 month-old children in northeast Brazil, the combination of MPO and neopterin added power in predicting subsequent growth impairment compared with MPO alone [64]. The clustering of biomarkers into an EED 'activity score' has also been used to explore associations with vaccine responses. In one study, the median EED 'activity score' was higher in Nicaraguan infants who did not seroconvert to RotaTeq compared with those who did (5.0 vs 3.5, respectively; $\mathrm{p}=0.03$ ) [51]. 
By contrast, there was no association between OPV3 seroconversion and this disease activity score in 293 Indian infants enrolled in the azithromycin trial [52]. It is worth noting that levels of intestinal inflammation measured in the Nicaraguan infants [51] were much lower on average compared with the studies from the Indian subcontinent. For example, among the 590 Bangladeshi infants in the PROVIDE study, levels of fecal calprotectin and MPO at 3 months of age were elevated in 82.7 and $88.1 \%$, respectively (using normal values based on Western standards) [56], highlighting the high prevalence of intestinal inflammation among young children in low-income countries.

Taken together, the evidence for intestinal inflammation altering oral vaccine responses is heterogeneous among the few available studies. The positive associations described in two studies between intestinal inflammation and vaccine response raise the possibility that an inflammatory intestinal milieu, perhaps counterintuitively, favors a robust immune response; however, this requires further evaluation.

\section{Domain 4 - microbial translocation \& immune activation}

There is a lack of reliable assays to detect microbial translocation. LPS (or endotoxin) is a key constituent in the membrane of Gram-negative bacteria, and therefore a plausible measure of translocation from the gut; however, it is difficult in young infants to avoid endotoxin contamination during venepuncture. Alternatively, IgM or $\operatorname{IgG}$ specific to the core domain of endotoxin (EndoCAb) is an indirect measure of LPS exposure; however, there have been technical problems with the commercially available assay [65]. Soluble CD14 (sCD14), which acts as a co-receptor in the LPS/TLR4 signal transduction pathway, is a marker of monocyte activation, particularly in response to endotoxin. Both EndoCAb and $\mathrm{sCD} 14$ have been reported in four studies, with conflicting results both within and between studies (Figure 2). In one cohort of Bangladeshi infants, sCD14 measured at 6 weeks of age was negatively associated with both OPV1 seroconversion and rotavirus protection but this association became positive at week 16 (for OPV1 only) [56]. In a separate cohort of older Bangladeshi children from the same urban slum region (Mirpur), sCD14 measured on the day of vaccine receipt was positively associated with Vibrio cholerae LPS, implying that microbial translocation may augment immune responses to the oral vaccine. However, in the same study, EndoCAb was negatively associated with cholera toxin-specific effector memory $\mathrm{T}$ cells suggesting that in the context of high microbial translocation, the T-cell response to oral cholera vaccine is attenuated [57]. In southern India, neither EndoCAb nor sCD14 were associated with OPV3 seroconversion or shedding [52]. The same was true in a Zambian study, where no association was seen for either EndoCAb or sCD14 and seroconversion to RVV [55].

Tryptophan is an aromatic amino acid, which has important anti-inflammatory effects via its catabolism to kynurenine. The enzyme indoleamine 2,3-dioxygenase 1 (IDO1) mediates this pathway and is expressed on both immune and epithelial cells [66]. Indoleamine 2,3-dioxygenase 1 activity, which is reflected in the kynurenine:tryptophan (KT) ratio, therefore modulates systemic and local immune responses [67] and has been used as a marker in systemic inflammatory conditions including inflammatory bowel disease and HIV [68-70]. It has also recently been explored as a candidate marker for EED. In a Peruvian birth cohort, the KT ratio not only correlated with linear growth and systemic markers of inflammation but was also predictive of failed response to OPV1 [53]. C-reactive protein is an acute phase protein synthesized by the liver in response to the proinflammatory cytokine IL-6. However, among Bangladeshi infants in the PROVIDE study, there was no clear association between a range of proinflammatory markers (IL1- $\beta$, IL-6 and C-reactive protein) and responses to OPV or RVV [56]; IL-10, which downregulates Th1 cytokines, when grouped into quartiles was negatively associated with rotavirus IgA seroconversion if in the 50th-75th percentile but positively correlated if in the 75th-100th percentile. Interestingly, a number of the systemic biomarkers were also positively associated with responses to the parenteral tetanus vaccine.

SIBO has been implicated in the development of EED [36]. Markers of EED such as fecal Reg1 $\beta$ and fecal calprotectin are elevated in SIBO-positive children [36]. The gold standard diagnosis of SIBO requires sampling and culture of fluid from the intestinal lumen, which is impractical in infants. Instead, testing the hydrogen content of exhaled air is preferred as a less invasive measure of SIBO, although low sensitivity and specificity have been reported [71]. Only one study has measured SIBO in the context of oral vaccine responses. This study found an association between SIBO (measured using hydrogen breath testing) and decreased immunogenicity of the oral cholera vaccine in a cohort of Chilean children [54], even though the prevalence of SIBO in the cohort (5.6\%) was lower than anticipated $(20 \%)$ and lower in prevalence than vaccine failures in these populations.

It is likely that the four domains of EED interact, as depicted in Figure 1. The tight grouping of EED biomarkers (at least one from each domain) in the cluster analysis described in the PROVIDE study supports this [72]. In the Nicaragua study, fecal biomarkers of intestinal inflammation (neopterin, MPO and calprotectin) were correlated 
with a fecal marker of permeability (AAT) [51]. In the Indian azithromycin trial, all fecal biomarkers (neopterin, $\mathrm{MPO}$, calprotectin and AAT) correlated with each other; however, they did not correlate with plasma biomarkers of intestinal epithelial damage (I-FABP) or microbial translocation (EndoCAb, sCD14) [52].

\section{Evidence from intervention studies}

Several studies have explored interventions to augment oral vaccine performance including micronutrients, antimicrobials, probiotics and dosing strategies [73]; however, we identified only one intervention trial that measured EED biomarkers. Some EED biomarkers (calprotectin, MPO and AAT) were reduced in children randomized to a 3-day course of $10 \mathrm{mg} / \mathrm{kg}$ azithromycin compared with placebo [52]; however, this difference did not translate into improvements in OPV3 seroconversion. Other studies have examined interventions targeting EED directly, such as nutrient supplementation [74-77], anti-inflammatory agents [78], rifaximin [79] and probiotics [80]. None of these studies included a measure of vaccine response as a secondary outcome.

\section{Evidence from animal studies}

Animal models have been used to explore the impact of several exposures on oral vaccine responses. Probiotic colonization, for example, protects against diarrhea after rotavirus challenge in gnotobiotic piglets [81]; and protein energy malnutrition has been shown to alter IgA responses to rotavirus vaccination in mice [82]. We found one animal study that explored the impact of EED on oral vaccine responses. In a gnotobiotic pig model, following three doses of oral RVV, more rotavirus-specific immune cells were detectable in pigs colonized with a healthy compared with unhealthy human gut microbiota [83]. The difference between 'healthy' and 'unhealthy' was based on two Nicaraguan infant donors with divergent EED scores and RotaTeq responses [51]; however, they failed to recapitulate the histological changes of enteropathy. A more robust model of EED was recently developed in mice, by introducing consumption of a low protein/fat diet alongside iterative oral exposure to commensal Bacteroidales species and Escherichia coli [31]. It would be valuable to further explore vaccine responses in this murine model of EED.

\section{Evidence from other gastrointestinal conditions}

EED has some phenotypic overlap with other chronic inflammatory conditions affecting the intestine, and we therefore reviewed studies that had evaluated oral vaccine performance in the context of chronic gastrointestinal disorders.

\section{Celiac disease}

The changes of EED are morphologically similar to those of celiac disease, an enteropathy resulting from exposure to the food protein gluten in genetically susceptible hosts. Antibody responses to parenteral vaccines are generally unaffected [84-87] as might be expected, since these vaccines bypass the gut. One study from the 1970's describes reduced neutralizing antibody titers to OPV in patients with celiac disease [88] which was attributed to IgA deficiency; however, a later study found no impairment in the magnitude of serum IgA or IgG responses to a monovalent OPV [89]. The relevance of these results for EED is unclear, given that abnormalities of the small intestinal mucosa resolve over time in celiac disease after introduction of gluten-free diets.

\section{Inflammatory bowel disease}

The inflammation of Crohn's disease is transmural and variably distributed along the entire length of the intestine, rather than the confluent small bowel distribution characteristic of EED. In a 1979 study, patients with Crohn's disease given a trial treatment of oral BCG demonstrated evidence of anergy [90]. However, we found no other studies examining the response to oral vaccination in IBD. Altered immune responses to parenteral vaccines have been described in IBD patients but the immune defects are attributed to immunosuppressive treatment rather than intestinal disease [91].

\section{Intestinal resection}

A dramatically shortened bowel following intestinal resection reduces the mucosal surface area and may affect uptake of oral vaccines. However, RotaTeq, given to infants with a history of bowel resection, was immunogenic despite varying lengths of residual bowel [92]. A study of adults with intestinal resection reported unaltered immune 
responses to oral cholera vaccine when compared with age-matched healthy volunteers [93], although all had undergone colectomies due to ulcerative colitis, and the impact of small intestinal resection was not evaluated.

\section{Evidence from conditions overlapping with EED}

Other perturbations in the intestinal milieu, overlapping and interacting with EED, may also contribute to the pathogenesis of impaired oral vaccine responses (Figure 1). First, diarrhea is common among infants in low-income countries and has been linked to reduced vaccine efficacy; OPV has consistently been shown to be less effective in children with concurrent diarrhea [94,95]. Diarrhea leads to shorter intestinal transit times, which may limit vaccine exposure. Increased mucosal innate immune responses may also impair vaccine replication and permeation. Second, enteropathogens may inhibit oral vaccines. Findings from a systematic review showed that concurrent enterovirus infection attenuates immune responses to OPV [96]. More recently, a study among Bangladeshi infants has shown that enterovirus infection at the time of vaccination impairs immune responses to RVV as well as OPV [97]. Finally, the microbiota has an important role in the development and maturation of the mucosal and systemic immune systems. A study in Ghana found differences in the composition of the bacterial microbiota between responders and nonresponders to oral RVV [98]. In prevaccination fecal specimens, increases in the Firmicutes phylum (particularly Streptococcus bovis) correlated with RVV seroconversion while an increased abundance of the Bacteroidetes phylum was associated with non-response. However, a recent study in Indian infants found no significant association between the bacterial microbiota composition and Rotarix seroconversion [99]. None of these studies measured EED and therefore it is difficult to determine whether vaccine response is shaped by these entities alone or through EED. However, data are beginning to emerge from larger cohorts such as the MAL-ED study [100], which may help to delineate these relationships.

\section{Discussion}

It is biologically plausible that EED contributes to oral vaccine failure. Broadly, it also seems that population trends in EED prevalence are inversely correlated with vaccine response. However, in our review of the literature, few consistent findings emerged from existing studies, which were highly heterogeneous. Many biomarkers did not correlate significantly with oral vaccine responses; where significant associations did exist, there were often conflicting findings both between and even within studies. It therefore remains uncertain whether EED contributes to oral vaccine failure based on current data, and further studies are required.

There are likely to be a number of reasons for the heterogeneous findings. First, there were problems with study design. Two studies [51,57] included fewer than 50 subjects and were likely underpowered to detect significant differences, although power calculations were not provided. Several studies measured multiple biomarkers, sometimes at several time points, and used varying analysis methods; these high dimensional data are prone to type 1 error. Second, exploring associations with oral vaccine performance is hampered by lack of a case definition of EED. Each study selected a different group of biomarkers to define EED. Two studies [51,52] reported EED using a score based on a combination of three fecal inflammatory markers, described elsewhere [63]. Grassly et al. coded EED as a binary variable, which was categorized as present if one or more biomarkers measurements were in the top quartile [52]; Becker-Dreps $e t$ al. added a further inflammatory biomarker to the score to make a '4 Biomarker EED score' [51]. Other studies used cluster analyses to highlight the most informative biomarkers [72]. Third, cross-comparison is further complicated by multiple differences between studies. For example, the characteristics of each oral vaccine differ, whether killed (oral cholera vaccines) or live (oral polio, rotavirus and typhoid vaccines) with varying strains, adjuvant properties and sites of replication (if live) in the intestinal tract. It is possible that each vaccine is influenced differently by the intestinal milieu. There are also differences in age across studies, ranging from 2 months to school age (Table 2). We believe that EED evolves over time, although we lack understanding of the exact kinetics; differences in age may be associated with substantial changes in gut architecture and function, capturing different windows in the progression of EED. Fourth, markers used to measure oral vaccine immunogenicity do not always correlate with protection from disease, which is the gold standard measure of efficacy. Rotavirus IgA seroconversion, for example, used as an outcome measure in four of the studies above, is likely a poor correlate of protection from rotavirus diarrhea in low-income countries [101]. However, measuring oral vaccine immunogenicity is often preferred to protective efficacy, which necessitates large numbers of study participants and detection of disease cases. Only one study, by Naylor et al., used Rotarix protection as an outcome by recording cases of rotavirus diarrhea in vaccinated infants up to 1 year of age [56]. Finally, all the studies were carried out within relatively 
homogeneous populations, often with ubiquitous EED; to date, an appropriate control group has therefore been lacking.

It is possible that the conflicting evidence arising from this small group of studies is because no biological association exists between oral vaccine underperformance and EED, and instead there are other explanations for why oral vaccines are less efficacious in low-income countries. It is also likely that biomarkers of EED are of limited accuracy. Nevertheless, the overlap between EED and oral vaccine underperformance is striking and the magnitude of intestinal damage when compared with children in high-income countries is high. In a Zimbabwean cohort for example, levels of I-FABP exceeded those of European children with celiac disease [102]. As our understanding of EED evolves, and the range of biomarkers available to characterize different domains of the condition expands, further well-designed studies are needed to investigate this hypothesis further.

\section{Conclusion \& future perspective}

Current evidence is insufficient to determine whether EED contributes to oral vaccine underperformance. Several factors complicating existing studies need to be resolved to clarify this important question. First, more studies are needed to inform a case definition of EED. A revised case definition has recently been proposed for use in therapeutic trials [48], which includes criteria akin to the Jones criteria for rheumatic fever, but it does not characterize all domains of the complex pathogenic pathway underlying EED [103]. The EED Biomarkers Initiative Consortium supported by the Bill \& Melinda Gates Foundation is evaluating several candidate biomarkers, and in some sites work is under way to correlate biomarkers with gut biopsies or confocal laser endomicroscopic findings. Newer biomarkers such as KT ratio and citrulline should be further investigated in different cohorts especially in the context of rotavirus vaccination; work in this field is currently underway in a cohort of Zimbabwean infants [58]. Second, studies should align their selection of biomarkers, measurement time points and outcomes, to facilitate cross-site comparisons. Methods that allow for high dimensional and correlated biomarker data need to be employed [104]. Finally, it is worth considering that different oral vaccines may be impacted by different factors. Conclusions drawn from OPV failure may not apply to RVV and vice versa. The most pressing of these at present is RVV underperformance and this should be the key focus of studies going forward, including efforts to find a better correlate of protection for RVV in low-income countries.

\section{Executive summary}

Oral vaccines have reduced immunogenicity and efficacy in low-income countries

- Oral vaccines are a vital tool for tackling enteric infections.

- However, oral rotavirus, polio, cholera and typhoid vaccines have consistently been shown to be less immunogenic and efficacious in low-income countries.

- A number of factors have been implicated but the precise reasons for underperformance remain unclear.

Environmental enteric dysfunction

- Environmental enteric dysfunction (EED) is a subclinical condition almost ubiquitous among children living in poverty, where oral vaccines underperform.

- It is characterized by villous blunting and mucosal inflammation as well as functional disturbances, which include increased intestinal permeability and absorptive capacity.

- The precise etiology remains unclear although microbial exposure appears to play a role.

- The definition and measurement of EED relies on noninvasive biomarkers, which capture the 'domains' of EED: intestinal permeability, intestinal epithelial damage, intestinal inflammation and microbial translocation.

EED \& oral vaccine failure

- We identified eight studies describing oral vaccine responses in the context of EED across five countries.

- Four studies reported a negative association between EED and oral vaccine responses; two studies showed no significant association; and two studies concluded that EED was positively correlated with immunogenicity to oral vaccine antigens.

- Across the four domains of EED few consistent trends emerged.

- Treatment of enteropathy has not resulted in improvements in oral vaccine immunogenicity to date.

- There is no clear evidence of altered oral vaccine responses in other gastrointestinal conditions.

Conclusion

- Existing studies exploring the relationship between EED and oral vaccine performance are few and highly heterogeneous.

- Although it is plausible that EED contributes to oral vaccine failure, the current evidence remains insufficient.

- Further well-designed studies are needed. 


\section{Supplementary data}

To view the supplementary data that accompany this paper please visit the journal website at: www.futuremedicine.com/doi/sup $\mathrm{pl} / 10.2217 / \mathrm{fmb}-0016-0016$

\section{Financial \& competing interests disclosure}

JAC (Grant 201293/Z/16/Z) and AJP (Grant 108065/Z/15/Z) are funded by the Wellcome Trust. JAC and AJP also received funding from DfID via the Sanitation \& Hygiene Applied Research for Equity (SHARE) Consortium. The authors have no other relevant affiliations or financial involvement with any organization or entity with a financial interest in or financial conflict with the subject matter or materials discussed in the manuscript apart from those disclosed.

No writing assistance was utilized in the production of this manuscript.

\section{Open access}

This article is distributed under the terms of the Creative Commons Attribution License 4.0 which permits any use, distribution, and reproduction in any medium, provided the original author(s) and the source are credited. To view a copy of the license, visit http://creativecommons.org/licenses/by/4.0/

\section{References}

Papers of special note have been highlighted as: $\bullet$ of interest; $\bullet \bullet$ of considerable interest

1. GBD Diarrhoeal Diseases Collaborators. Estimates of global, regional, and national morbidity, mortality, and aetiologies of diarrhoeal diseases: a systematic analysis for the Global Burden of Disease Study 2015. Lancet Infect. Dis. 17(9), 909-948 (2017).

2. Liu L, Oza S, Hogan D et al. Global, regional, and national causes of child mortality in 2000-13, with projections to inform post-2015 priorities: an updated systematic analysis. Lancet 385(9966), 430-440 (2015).

3. GBD 2016 Disease and Injury Incidence and Prevalence Collaborators Global, regional, and national incidence, prevalence, and years lived with disability for 328 diseases and injuries for 195 countries, 1990-2016: a systematic analysis for the Global Burden of Disease Study 2016. Lancet 390(10100), 1211-1259 (2017).

4. VIEW-hub. Interactive mapping tool. www.view-hub.org/viz/

5. Patel M, Shane AL, Parashar UD, Jiang B, Gentsch JR, Glass RI. Oral rotavirus vaccines: how well will they work where they are needed most? J. Infect. Dis. 200(Suppl. 1), S39-S48 (2009).

6. Plotkin SA, Lebrun A, Koprowski H. Vaccination with the CHAT strain of type 1 attenuated poliomyelitis virus in Leopoldville. Belgian Congo. 2. Studies of the safety and efficacy of vaccination. Bull. World Health Organ. 22, 215-234 (1960).

7. Patriarca PA, Wright PF, John TJ. Factors affecting the immunogenicity of oral poliovirus vaccine in developing countries: review. Rev. Infect. Dis. 13(5), 926-939 (1991).

8. Hallander HO, Paniagua M, Espinoza F et al. Calibrated serological techniques demonstrate significant different serum response rates to an oral killed cholera vaccine between Swedish and Nicaraguan children. Vaccine 21(1-2), 138-145 (2002).

9. Armah GE, Sow SO, Breiman RF et al. Efficacy of pentavalent rotavirus vaccine against severe rotavirus gastroenteritis in infants in developing countries in sub-Saharan Africa: a randomised, double-blind, placebo-controlled trial. Lancet 376(9741), 606-614 (2010).

-• Efficacy trial demonstrating the underperformance of oral rotavirus vaccine across three low income countries (Ghana, Kenya and Mali).

10. Zaman K, Dang DA, Victor JC et al. Efficacy of pentavalent rotavirus vaccine against severe rotavirus gastroenteritis in infants in developing countries in Asia: a randomised, double-blind, placebo-controlled trial. Lancet 376(9741), 615-623 (2010).

11. Ruiz-Palacios GM, Perez-Schael I, Velazquez FR et al. Safety and efficacy of an attenuated vaccine against severe rotavirus gastroenteritis. N. Engl. J. Med. 354(1), 11-22 (2006).

12. Vesikari T, Matson DO, Dennehy $\mathrm{P}$ et al. Safety and efficacy of a pentavalent human-bovine (WC3) reassortant rotavirus vaccine. $N$. Engl. J. Med. 354(1), 23-33 (2006).

13. Parker EP, Ramani S, Lopman BA et al. Causes of impaired oral vaccine efficacy in developing countries. Future Microbiol. 13, 97-118 (2017).

-• Review summarising the risk factors for oral vaccine failure and the heterogeneous nature of the evidence available.

14. Moon SS, Tate JE, Ray P et al. Differential profiles and inhibitory effect on rotavirus vaccines of nonantibody components in breast milk from mothers in developing and developed countries. Pediatr. Infect. Dis. J. 32(8), 863-870 (2013).

15. Platts-Mills JA, Babji S, Bodhidatta L et al. Pathogen-specific burdens of community diarrhoea in developing countries: a multisite birth cohort study (MAL-ED). Lancet Glob. Health 3(9), e564-e575 (2015).

16. Bucardo F, Nordgren J, Reyes Y, Gonzalez F, Sharma S, Svensson L. The Lewis A phenotype is a restriction factor for Rotateq and Rotarix vaccine-take in Nicaraguan children. Sci. Rep. 8(1), 1502 (2018). 
17. Black RE, Victora CG, Walker SP et al. Maternal and child undernutrition and overweight in low-income and middle-income countries. Lancet 382(9890), 427-451 (2013).

18. Smith MI, Yatsunenko T, Manary MJ et al. Gut microbiomes of Malawian twin pairs discordant for kwashiorkor. Science 339(6119), 548-554 (2013).

19. Smith LE, Stoltzfus RJ, Prendergast A. Food chain mycotoxin exposure, gut health, and impaired growth: a conceptual framework. Adv. Nutr. 3(4), 526-531 (2012).

20. Prendergast A, Kelly P. Enteropathies in the developing world: neglected effects on global health. Am. J. Trop. Med. Hyg. 86(5), 756-763 (2012).

21. Campbell DI, Murch SH, Elia M et al. Chronic T cell-mediated enteropathy in rural west African children: relationship with nutritional status and small bowel function. Pediatr. Res. 54(3), 306-311 (2003).

- Highlights the potential clinical importance of impaired gut barrier function in mediating stunting in infants in developing countries.

22. Veitch AM, Kelly P, Zulu IS, Segal I, Farthing MJ. Tropical enteropathy: a T-cell-mediated crypt hyperplastic enteropathy. Eur. J. Gastroenterol. Hepatol. 13(10), 1175-1181 (2001).

23. Brewster DR, Manary MJ, Menzies IS, O’loughlin EV, Henry RL. Intestinal permeability in kwashiorkor. Arch. Dis. Child. 76(3), 236-241 (1997).

24. Campbell DI, Elia M, Lunn PG. Growth faltering in rural Gambian infants is associated with impaired small intestinal barrier function, leading to endotoxemia and systemic inflammation. J. Nutr. 133(5), 1332-1338 (2003).

25. Cook GC. Aetiology and pathogenesis of postinfective tropical malabsorption (tropical sprue). Lancet 1(8379), 721-723 (1984).

26. Lindenbaum J, Alam AK, Kent TH. Subclinical small-intestinal disease in East Pakistan. Br. Med. J. 2(5530), 1616-1619 (1966).

27. Chacko CJ, Paulson KA, Mathan VI, Baker SJ. The villus architecture of the small intestine in the tropics: a necropsy study. J. Pathol. 98(2), 146-151 (1969).

28. Gerson CD, Kent TH, Saha JR, Siddiqi N, Lindenbaum J. Recovery of small-intestinal structure and function after residence in the tropics. II. Studies in Indians and Pakistanis living in New York City. Ann. Intern. Med. 75(1), 41-48 (1971).

29. Lindenbaum J, Gerson CD, Kent TH. Recovery of small-intestinal structure and function after residence in the tropics. I. Studies in Peace Corps volunteers. Ann. Intern. Med. 74(2), 218-222 (1971).

30. Lin A, Arnold BF, Afreen S et al. Household environmental conditions are associated with enteropathy and impaired growth in rural Bangladesh. Am. J. Trop. Med. Hyg. 89(1), 130-137 (2013).

31. Brown EM, Wlodarska M, Willing BP et al. Diet and specific microbial exposure trigger features of environmental enteropathy in a novel murine model. Nat. Commun. 6, 7806 (2015).

- The first animal model of environmental enteric dysfunction (EED) which showed the importance of undernutrition and specific microbial exposure in recapitulating the intestinal pathology found in humans.

32. Steiner TS, Lima AA, Nataro JP, Guerrant RL. Enteroaggregative Escherichia coli produce intestinal inflammation and growth impairment and cause interleukin-8 release from intestinal epithelial cells. J. Infect. Dis. 177(1), 88-96 (1998).

33. Opintan JA, Newman MJ, Ayeh-Kumi PF et al. Pediatric diarrhea in southern Ghana: etiology and association with intestinal inflammation and malnutrition. Am. J. Trop. Med. Hyg. 83(4), 936-943 (2010).

34. Roy SK, Behrens RH, Haider R et al. Impact of zinc supplementation on intestinal permeability in Bangladeshi children with acute diarrhoea and persistent diarrhoea syndrome. J. Pediatr. Gastroenterol. Nutr. 15(3), 289-296 (1992).

35. Goto R, Panter-Brick C, Northrop-Clewes CA, Manahdhar R, Tuladhar NR. Poor intestinal permeability in mildly stunted Nepali children: associations with weaning practices and Giardia lamblia infection. Br. J. Nutr. 88(2), 141-149 (2002).

36. Donowitz JR, Haque R, Kirkpatrick BD et al. Small intestine bacterial overgrowth and environmental enteropathy in Bangladeshi children. mBio 7(1), e02102-e02115 (2016).

37. Fagundes Neto U, Martins MC, Lima FL, Patricio FR, Toledo MR. Asymptomatic environmental enteropathy among slum-dwelling infants. J. Am. Coll. Nutr. 13(1), 51-56 (1994).

38. Ordiz MI, Stephenson K, Agapova $S$ et al. Environmental enteric dysfunction and the fecal microbiota in Malawian children. Am. J. Trop. Med. Hyg. 96(2), 473-476 (2017).

39. Keusch GT, Denno DM, Black RE et al. Environmental enteric dysfunction: pathogenesis, diagnosis, and clinical consequences. Clin. Infect. Dis. 59(Suppl. 4), S207-S212 (2014).

- Historical review of EED including description of four categories of aberrant gut structure and function.

40. Kelly P, Menzies I, Crane R et al. Responses of small intestinal architecture and function over time to environmental factors in a tropical population. Am. J. Trop. Med. Hyg. 70(4), 412-419 (2004).

- Evaluates mucosal architecture and function longitudinally and described associations with stool microbiology and environmental factors. 
41. Papadia C, Kelly P, Caini S et al. Plasma citrulline as a quantitative biomarker of HIV-associated villous atrophy in a tropical enteropathy population. Clin. Nutr. 29(6), 795-800 (2010).

42. Korpe PS, Petri WA Jr. Environmental enteropathy: critical implications of a poorly understood condition. Trends Mol. Med. 18(6), 328-336 (2012).

43. Serazin AC, Shackelton LA, Wilson C, Bhan MK. Improving the performance of enteric vaccines in the developing world. Nat. Immunol. 11(9), 769-773 (2010).

44. Patel M, Glass RI, Jiang B, Santosham M, Lopman B, Parashar U. A systematic review of anti-rotavirus serum IgA antibody titer as a potential correlate of rotavirus vaccine efficacy. J. Infect. Dis. 208(2), 284-294 (2013).

45. Deming MS, Linkins RW, Jaiteh KO, Hull HF. The clinical efficacy of trivalent oral polio vaccine in The Gambia by season of vaccine administration. J. Infect. Dis. 175(Suppl. 1), S254-S257 (1997).

46. Keusch GT, Rosenberg IH, Denno DM et al. Implications of acquired environmental enteric dysfunction for growth and stunting in infants and children living in low- and middle-income countries. Food Nutr. Bull. 34(3), 357-364 (2013).

47. Petri WA Jr, Naylor C, Haque R. Environmental enteropathy and malnutrition: do we know enough to intervene? BMC Med. 12, 187 (2014).

48. Denno DM, Tarr PI, Nataro JP. Environmental enteric dysfunction: a case definition for intervention trials. Am. J. Trop. Med. Hyg. 97(6), 1643-1646 (2017).

49. Levine MM. Immunogenicity and efficacy of oral vaccines in developing countries: lessons from a live cholera vaccine. BMC Biol. 8,129 (2010).

50. Gilmartin AA, Petri WA Jr. Exploring the role of environmental enteropathy in malnutrition, infant development and oral vaccine response. Philos. Trans. R. Soc. Lond. B Biol. Sci. 370(1671), pii:20140143 (2015).

51. Becker-Dreps $S$, Vilchez $S$, Bucardo $\mathrm{F}$ et al. The association between fecal biomarkers of environmental enteropathy and rotavirus vaccine response in Nicaraguan infants. Pediatr. Infect. Dis. J. 36(4), 412-416 (2017).

-• Evaluates immune responses to oral rotavirus vaccine in Nicaraguan infants with EED.

52. Grassly NC, Praharaj I, Babji S et al. The effect of azithromycin on the immunogenicity of oral poliovirus vaccine: a double-blind randomised placebo-controlled trial in seronegative Indian infants. Lancet Infect. Dis. 16(8), 905-914 (2016).

-. In this randomized placebo controlled trial, azithromycin had no impact on oral polio vaccine immunogenicity but did reduce biomarkers of EED.

53. Kosek MN, Mduma E, Kosek PS et al. Plasma tryptophan and the kynurenine-tryptophan ratio are associated with the acquisition of statural growth deficits and oral vaccine underperformance in populations with environmental enteropathy. Am. J. Trop. Med. Hyg. 95(4), 928-937 (2016).

-. Evaluates immune responses to oral polio vaccine in Peruvian infants with EED, employing two novel biomarkers of EED.

54. Lagos R, Fasano A, Wasserman SS et al. Effect of small bowel bacterial overgrowth on the immunogenicity of single-dose live oral cholera vaccine CVD 103-HgR. J. Infect. Dis. 180(5), 1709-1712 (1999).

55. Mwape I, Bosomprah S, Mwaba J et al. Immunogenicity of rotavirus vaccine (RotarixTM) in infants with environmental enteric dysfunction. PLoS ONE 12(12), e0187761 (2017).

- Evaluates immune responses to oral rotavirus vaccine in Zambian children with EED.

56. Naylor C, Lu M, Haque R et al. Environmental enteropathy, oral vaccine failure and growth faltering in infants in Bangladesh. EBioMedicine 2(11), 1759-1766 (2015).

-• Evaluates immune responses to oral polio and oral rotavirus vaccines in Bangladeshi infants with EED.

57. Uddin MI, Islam S, Nishat NS et al. Biomarkers of environmental enteropathy are positively associated with immune responses to an oral cholera vaccine in Bangladeshi children. PLoS Negl. Trop. Dis. 10(11), e0005039 (2016)

-• Evaluates immune responses to oral cholera vaccine in Bangladeshi children with EED.

58. Prendergast AJ, Humphrey JH, Mutasa $\mathrm{K}$ et al. Assessment of environmental enteric dysfunction in the SHINE trial: methods and challenges. Clin. Infect. Dis. 61(Suppl. 7), S726-S732 (2015).

59. Peterson KM, Guo X, Elkahloun AG et al. The expression of REG 1A and REG $1 \mathrm{~B}$ is increased during acute amebic colitis. Parasitol. Int. 60(3), 296-300 (2011).

60. Granlund A, Beisvag V, Torp SH et al. Activation of REG family proteins in colitis. Scand. J. Gastroenterol. 46(11), 1316-1323 (2011).

61. Crenn P, Messing B, Cynober L. Citrulline as a biomarker of intestinal failure due to enterocyte mass reduction. Clin. Nutr. 27(3), 328-339 (2008).

62. Prata MM, Havt A, Bolick DT, Pinkerton R, Lima A, Guerrant RL. Comparisons between myeloperoxidase, lactoferrin, calprotectin and lipocalin-2, as fecal biomarkers of intestinal inflammation in malnourished children. J. Transl. Sci. 2(2), 134-139 (2016).

63. Kosek M, Haque R, Lima A et al. Fecal markers of intestinal inflammation and permeability associated with the subsequent acquisition of linear growth deficits in infants. Am. J. Trop. Med. Hyg. 88(2), 390-396 (2013). 
64. Guerrant RL, Leite AM, Pinkerton R et al. Biomarkers of environmental enteropathy, inflammation, stunting, and impaired growth in children in northeast Brazil. PLoS ONE 11(9), e0158772 (2016).

65. Kosek M, Guerrant RL, Kang G et al. Assessment of environmental enteropathy in the MAL-ED cohort study: theoretical and analytic framework. Clin. Infect. Dis. 59(Suppl. 4), S239-S247 (2014).

66. Richard DM, Dawes MA, Mathias CW, Acheson A, Hill-Kapturczak N, Dougherty DM. L-tryptophan: basic metabolic functions, behavioral research and therapeutic indications. Int. J. Tryptophan. Res. 2, 45-60 (2009).

67. Ciorba MA. Indoleamine 2,3 dioxygenase in intestinal disease. Curr. Opin. Gastroenterol. 29(2), 146-152 (2013).

68. Gupta NK, Thaker AI, Kanuri N et al. Serum analysis of tryptophan catabolism pathway: correlation with Crohn's disease activity. Inflamm. Bowel Dis. 18(7), 1214-1220 (2012).

69. Lee $\mathrm{S}$, Byakwaga $\mathrm{H}$, Boum $\mathrm{Y}$ et al. Immunologic pathways that predict mortality in HIV-infected Ugandans initiating ART. J. Infect. Dis. 215(8), 1270-1274 (2017).

70. Nikolaus S, Schulte B, Al-Massad N et al. Increased tryptophan metabolism is associated with activity of inflammatory bowel diseases. Gastroenterology 153(6), 1504.e2-1516.e2 (2017).

71. Riordan SM, Mciver CJ, Walker BM, Duncombe VM, Bolin TD, Thomas MC. The lactulose breath hydrogen test and small intestinal bacterial overgrowth. Am. J. Gastroenterol. 91(9), 1795-1803 (1996).

72. Naylor $\mathrm{C}, \mathrm{Lu} \mathrm{M}$, Haque $\mathrm{R}$ et al. Environmental enteropathy, oral vaccine failure and growth faltering in infants in Bangladesh. EBioMedicine 2(11), 1759-1766 (2015).

73. Church JPE, Kirkpatrick B, Grassly N, Prendergast AJ. Interventions to improve oral vaccine performance in developing countries: protocol for a systematic review and meta-analysis. (2017). www.crd.york.ac.uk/PROSPEROFILES/60608_PROTOCOL_20180108.pdf

74. Louis-Auguste J, Greenwald S, Simuyandi M, Soko R, Banda R, Kelly P. High dose multiple micronutrient supplementation improves villous morphology in environmental enteropathy without HIV enteropathy: results from a double-blind randomised placebo controlled trial in Zambian adults. BMC Gastroenterol. 14, 15 (2014).

75. Smith HE, Ryan KN, Stephenson KB et al. Multiple micronutrient supplementation transiently ameliorates environmental enteropathy in Malawian children aged 12-35 months in a randomized controlled clinical trial. J. Nutr. 144(12), 2059-2065 (2014).

76. Wang AZ, Shulman RJ, Crocker AH et al. A combined intervention of zinc, multiple micronutrients, and albendazole does not ameliorate environmental enteric dysfunction or stunting in rural malawian children in a double-blind randomized controlled trial. $J$. Nutr. 147(1), 97-103 (2017).

77. Trehan I, Benzoni NS, Wang AZ et al. Common beans and cowpeas as complementary foods to reduce environmental enteric dysfunction and stunting in Malawian children: study protocol for two randomized controlled trials. Trials 16, 520 (2015).

78. Jones KD, Hunten-Kirsch B, Laving AM et al. Mesalazine in the initial management of severely acutely malnourished children with environmental enteric dysfunction: a pilot randomized controlled trial. BMC Med. 12, 133 (2014).

79. Trehan I, Shulman RJ, Ou CN, Maleta K, Manary MJ. A randomized, double-blind, placebo-controlled trial of rifaximin, a nonabsorbable antibiotic, in the treatment of tropical enteropathy. Am. J. Gastroenterol. 104(9), 2326-2333 (2009).

80. Galpin L, Manary MJ, Fleming K, Ou CN, Ashorn P, Shulman RJ. Effect of lactobacillus GG on intestinal integrity in Malawian children at risk of tropical enteropathy. Am. J. Clin. Nutr. 82(5), 1040-1045 (2005).

81. Chattha KS, Vlasova AN, Kandasamy S, Rajashekara G, Saif LJ. Divergent immunomodulating effects of probiotics on T cell responses to oral attenuated human rotavirus vaccine and virulent human rotavirus infection in a neonatal gnotobiotic piglet disease model. $J$. Immunol. 191(5), 2446-2456 (2013).

82. Maier EA, Weage KJ, Guedes MM et al. Protein-energy malnutrition alters IgA responses to rotavirus vaccination and infection but does not impair vaccine efficacy in mice. Vaccine 32(1), 48-53 (2013).

83. Twitchell EL, Tin C, Wen K et al. Modeling human enteric dysbiosis and rotavirus immunity in gnotobiotic pigs. Gut Pathog. 8, 51 (2016).

84. Mckinley M, Leibowitz S, Bronzo R, Zanzi I, Weissman G, Schiffman G. Appropriate response to pneumococcal vaccine in celiac sprue. J. Clin. Gastroenterol. 20(2), 113-116 (1995).

85. Sari S, Dalgic B, Basturk B, Gonen S, Soylemezoglu O. Immunogenicity of hepatitis A vaccine in children with celiac disease. J. Pediatr. Gastroenterol. Nutr. 53(5), 532-535 (2011).

86. Schappi MG, Meier S, Bel M, Siegrist CA, Posfay-Barbe KM, Group HNS. Protective antibody responses to influenza A/H1N1/09 vaccination in children with celiac disease. J. Pediatr. Gastroenterol. Nutr. 54(6), 817-819 (2012).

87. Opri R, Veneri D, Mengoli C, Zanoni G. Immune response to Hepatitis B vaccine in patients with celiac disease: a systematic review and meta-analysis. Hum. Vaccin. Immunother. 11(12), 2800-2805 (2015).

88. Beale AJ, Douglas AP, Parish WE, Hobbs JR. Impaired IgA responses in coeliac disease. Lancet 1(7711), 1198-1200 (1971).

89. Mawhinney $\mathrm{H}$, Love $\mathrm{AH}$. The immunoglobulin class responses to oral poliovaccine in coeliac disease. Clin. Exp. Immunol. 21(3), 399-406 (1975). 
90. Burnham WR, Lennard-Jones JE, Hecketsweiler P, Colin R, Geffroy Y. Oral BCG vaccine in Crohn's disease. Gut 20(3), 229-233 (1979).

91. Nguyen DL, Nguyen ET, Bechtold ML. Effect of immunosuppressive therapies for the treatment of inflammatory bowel disease on response to routine vaccinations: a meta-analysis. Dig. Dis. Sci. 60(8), 2446-2453 (2015).

92. Mcgrath EJ, Thomas R, Duggan C, Asmar BI. Pentavalent rotavirus vaccine in infants with surgical gastrointestinal disease. J. Pediatr. Gastroenterol. Nutr. 59(1), 44-48 (2014).

93. Kilhamn J, Brevinge H, Svennerholm AM, Jertborn M. Immune responses in ileostomy fluid and serum after oral cholera vaccination of patients colectomized because of ulcerative colitis. Infect. Immun. 66(8), 3995-3999 (1998).

94. Factors affecting the immunogenicity of oral poliovirus vaccine: a prospective evaluation in Brazil and the Gambia. World Health Organization Collaborative Study Group on Oral Poliovirus Vaccine. J. Infect. Dis. 171(5), 1097-1106 (1995).

95. Cardemil CV, Estivariz C, Shrestha L et al. The effect of diarrheal disease on bivalent oral polio vaccine (bOPV) immune response in infants in Nepal. Vaccine 34(22), 2519-2526 (2016).

96. Parker EP, Kampmann B, Kang G, Grassly NC. Influence of enteric infections on response to oral poliovirus vaccine: a systematic review and meta-analysis. J. Infect. Dis. 210(6), 853-864 (2014).

97. Taniuchi M, Platts-Mills JA, Begum S et al. Impact of enterovirus and other enteric pathogens on oral polio and rotavirus vaccine performance in Bangladeshi infants. Vaccine 34(27), 3068-3075 (2016).

98. Harris VC, Armah G, Fuentes $\mathrm{S}$ et al. Significant correlation between the infant gut microbiome and rotavirus vaccine response in rural Ghana. J. Infect. Dis. 215(1), 34-41 (2017).

99. Parker EPK, Praharaj I, Zekavati A et al. Influence of the intestinal microbiota on the immunogenicity of oral rotavirus vaccine given to infants in south India. Vaccine 36(2), 264-272 (2017).

100. Mccormick BJ, Lee GO, Seidman JC et al. Dynamics and trends in fecal biomarkers of gut function in children from 1-24 months in the MAL-ED study. Am. J. Trop. Med. Hyg. 96(2), 465-472 (2017).

101. Angel J, Steele AD, Franco MA. Correlates of protection for rotavirus vaccines: possible alternative trial endpoints, opportunities, and challenges. Hum. Vaccin. Immunother. 10(12), 3659-3671 (2014).

102. Prendergast AJ, Rukobo S, Chasekwa B et al. Stunting is characterized by chronic inflammation in Zimbabwean infants. PLoS ONE 9(2), e86928 (2014).

103. Owino V, Ahmed T, Freemark M et al. Environmental enteric dysfunction and growth failure/stunting in global child health. Pediatrics 138(6), pii:e20160641 (2016).

104. Lu M, Zhou J, Naylor C et al. Application of penalized linear regression methods to the selection of environmental enteropathy biomarkers. Biomark. Res. 5, 9 (2017). 